\title{
Die irische Parlamentswahl vom 25. Februar 2011: Ende des dominierten Parteiensystems?
}

\author{
Thomas Krumm
}

Nach der Erosion der beiden Volksparteien in Großbritannien und der erstmaligen Bildung einer Koalitionsregierung seit dem Zweiten Weltkrieg auf der Nachbarinsel im Mai 2010 zeichnet sich nun auch in Irland ein struktureller Umbruch des Parteiensystems ab. Die Immobilien- und Bankenkrise hat die dominierende Partei, Fianna Fáil, nach dreizehn Jahren und acht Monaten ununterbrochenen Regierens so schwer getroffen, dass eine schnelle Erholung wenig wahrscheinlich erscheint. Die Partei, deren Stimmenanteil bislang nie unter 39 Prozent gefallen war, erhielt bei der vorgezogenen Neuwahl des Dáil Éireann am 25. Februar 2011 nur noch 17,36 Prozent der Erstpräferenzen. Aufgrund des Disproportionalitätseffektes im Wahlrecht reichte dies nur für zwölf Prozent der Sitze. Fianna Fáil, seit 1927 im Dáil vertreten, hat seit ihrer erstmaligen Regierungsbeteiligung 1932 für über 61 Jahre den Premierminister (Taoiseach) beziehungsweise dessen Vorgänger, den Vorsitzenden des Exekutivrates, gestellt, die konkurrierende Fine Gael nur für knapp 17 Jahre. Das entspricht einem Anteil von über 78 Prozent für Fianna Fáil und knapp 22 Prozent für Fine Gael. ${ }^{1}$ Man kann also durchaus von einem dominierten Mehrparteiensystem sprechen, bei dem eine Partei über lange Zeit alle anderen stark überragt ${ }^{2}$, obwohl das irische Wahlrecht als eine Variante der Verhältniswahl einem solchen Systemtyp eigentlich keinen Vorschub leisten sollte. ${ }^{3}$ Die vorgezogene Parlamentswahl 2011 führte durch einen externen Schock - die globale Finanz- und Wirtschaftskrise hat Irland besonders hart getroffen - und internen Verschleiß der seit 1997 regierenden Fianna Fáil zu einem historisch einmaligen Verlust an Unterstützung dieser strukturellen Mehrheitspartei ${ }^{4}$ und zur erstmaligen Bildung einer Großen Koalition aus Fine Gael und Labour Party.

1 Vgl. Maura Adshead / Jonathan Tonge, Politics in Ireland, Basingstoke 2009, S. 88 ff. für Erklärungsansätze der Fianna Fáil-Dominanz im irischen Parteiensystem. Cum grano salis lässt sich Fianna Fáil als eine eher republikanisch-nationalistische, Fine Gael als eine eher christdemokratisch-konservative Partei beschreiben, John Coakley (The election and the party system, in: Michael Gallagher / Michael Marsh / Paul Mitchell (Hrsg.), How Ireland Voted 2002, Basingstoke 2003, S. 230 - 246) spricht von „Europe's most consistently successful vote-getter“ (S. 239).

2 Vgl. Giovanni Sartori, Parties and Party Systems. A Framework for Analysis, Cambridge 1976; Thomas Saalfeld, Parteien und Wahlen, Baden-Baden 2007, S. 184.

3 Vgl. Dieter Nohlen, Wahlrecht und Parteiensystem, Opladen 2009, S. 156. Umso erstaunlicher ist der hohe Anteil (54,5 Prozent) von Einparteiregierungen (einschließlich Minderheitsregierungen) zwischen 1945 und 1999 bei einer durchschnittlichen Zahl von 1,64 Regierungsparteien, vgl. Thomas Saalfeld, a.a.O. (Fn. 2), S. 206.

4 Nach der sozioökonomischen Theorie exogener Schocks ist davon auszugehen, dass sich die Staatsausgaben nach der Abarbeitung der Störung durch die Politik nicht wieder an das Vorkrisenniveau annähern, vgl. zum Beispiel Uwe Wagschal, Entwicklung, Determinanten und Vergleich der Staatsfinanzen, in: Manfred G. Schmidt / Reimut Zohlnhöfer, Regieren in der Bundesrepublik Deutschland, Wiesbaden 2006, S. 57 - 86, S. 69; ders., Bestimmungsfaktoren der Steuerpolitik, in: Manfred G. Schmidt (Hrsg.), Der Wohlfahrtsstaat, Wiesbaden 2007, S. 326 - 352, S. 339 . 


\section{Die Regierungskrise 2010}

Am 6. Mai 2008 trat Bertie Ahern nach fast elf Jahren als Premierminister wegen nichtdeklarierter Spendenannahmen während seiner Zeit als Finanzminister Anfang der 1990er Jahre zurück. Sein Nachfolger, Finanzminister Brian Cowen, agierte eher glücklos, besonders in den Verhandlungen mit EU und IWF über das irische Bankenrettungspaket vom Mai 2010, und wurde sogar von seinem Amtsvorgänger offen kritisiert - ein Novum in der irischen Geschichte. Dass die traditionelle Regierungspartei Fianna Fáil das von EU und IWF im Rahmen des so genannten Euro-Rettungsschirmes ${ }^{5}$ vorbereitete Finanzrettungspaket mit einem Umfang von 85 Milliarden Euro im Herbst 2010 akzeptierte, wenn auch widerwillig und nach längerem Zögern, wurde in der Öffentlichkeit weitgehend als Offenbarungseid der Regierung Cowen verstanden. ${ }^{6}$ Im Zuge der Banken- und Finanzkrise stieg die Staatsverschuldung von 25 Prozent des Bruttoinlandsprodukts (BIP) im Jahre 2007 auf 65,5 Prozent 2009 (EU 27: 74 Prozent). Während das BIP im Jahr 2000 um zehn Prozent gewachsen war, fiel es 2009 um 7,6 Prozent (EU 27: -4,2 Prozent); das Finanzierungsdefizit des Haushalts erreichte 2009 -14,4 Prozent (EU 27: -6,8 Prozent), und die Arbeitslosenquote stieg 2010 auf fast 14 Prozent. $^{7}$

Als in der zweiten Jahreshälfte 2010 die Probleme irischer Banken immer offensichtlicher wurden, drängten EU und IWF die Regierung, Kredite aus dem „Rettungsschirm“ anzunehmen, um die irischen Banken zu stabilisieren und eine Ausweitung der Krise auf weitere europäische Länder zu verhindern. Dieser Schritt mit den damit verbundenen Auflagen war in der Innenpolitik sehr unpopulär und motivierte die Oppositionsparteien und den kleinen Koalitionspartner von Fianna Fáil, die Grünen, baldige Neuwahlen zu fordern. Diese wurden von Taoiseach Cowen auch für Anfang 2011, nach Abschluss des Haushaltes, zugesagt. Um ihrer Forderung Nachdruck zu verleihen, zogen sich die Grünen am 23. Januar 2011 aus der Regierung zurück, trugen aber den neuen Haushalt noch mit. Am 1. Februar wurde der Dáil schließlich aufgelöst und am 25. Februar Neuwahlen abgehalten, die für Fianna Fáil das schlechteste Ergebnis ihrer Geschichte brachten und erstmals zu einer Großen Koalition führten.

5 Darunter ist die im Juni 2010 gegründete EFSF (Europäische Finanzstabilisierungsfazilität) gemeint, die aufgrund guter Bonität der sie tragenden starken Euro-Länder zinsgünstigere Kredite an die Krisenländer weitergeben kann, als dies durch den Markt möglich wäre. An das mögliche Kreditvolumen des EFSF von insgesamt 440 Milliarden Euro sind weitere 250 Milliarden Euro des IWF gekoppelt. Der deutsche Garantieanteil des am 29. Oktober vom Bundestag beschlossenen EFSF liegt bei 211 Milliarden Euro. Als Gegenleistung für die zinsgünstigeren EFSF-Kredite müssen die Krisenländer einen finanz- und wirtschaftspolitischen Konsolidierungsplan vorlegen beziehungsweise aushandeln, der schließlich einstimmig von den Finanzministern der EFSFStaaten akzeptiert werden muss. Bis Mitte 2013 soll die EFSF in einen ständigen Krisenmechanismus (ESM) überführt und der Lissabon-Vertrag (Art. 136) entsprechend geändert werden. Strittig ist noch die Frage, in welchem Umfang auch private Gläubiger der betroffenen Banken zum Beispiel durch Abschreibung ihrer Forderungen beteiligt werden sollen. Dies würde einerseits das Risiko für die Steuerzahler reduzieren, andererseits die Refinanzierung der Banken erschweren (vgl. Marcus Theurer / Stefan Ruhkamp, Irischen Banken fehlen 24 Milliarden Euro, in: FAZ vom 1. April 2011, S. 11).

6 Vgl. Johannes Leithäuser, Erneuerung auf der Oppositionsbank, in: FAZ vom 25. Februar 2011, S. 4.

7 Alle Angaben von Eurostat, http://epp.eurostat.ec.europa.eu/ (Abruf am 28. Februar 2011). 
Das mit EU und IWF vereinbarte Rettungspaket mit einer Kreditlinie von 85 Milliarden Euro, von denen 35 Milliarden von den irischen Banken abgerufen werden können, sowie die daran geknüpfte strenge Haushaltskonsolidierung waren neben der Rolle, die die irische Sprache in den Schulen spielen soll, die Hauptthemen im Wahlkampf. Der spätere Regierungschef Enda Kenny hatte die Sparpolitik und das Rettungspaket sowie den damit verbundenen Zeitplan im Wahlkampf zwar grundsätzlich akzeptiert, setzte sich jedoch auch für Nachverhandlungen bei den Zinsraten ein, die mit durchschnittlich 5,8 Prozent für Anleihen bis zu zehn Jahren als zu hoch empfunden wurden. Auf grundsätzliche Ablehnung traf das EU-Rettungspaket bei Sinn Féin und bei vielen unabhängigen Kandidaten. Fianna Fáil war in den Umfragen als Partei zwar abgestürzt, jedoch genoss der neue Vorsitzende Micheál Martin großes Vertrauen in der Bevölkerung. In Vorwahlumfragen erhielt er hohe Zustimmungsraten als „fähigster Kandidat“, gefolgt von Labourchef Eamon Gilmore und dann erst Fine Gael Vorsitzender Kenny.

Durch den Rettungspakt und den noch Ende 2010 von der scheidenden Fianna FáilGrüne-Koalition verabschiedeten Haushalt 2011 ist der Handlungsspielraum der neuen Regierung extrem eingeschränkt. Ihr sind Prioritäten extern vorgegeben: Abbau des enormen Haushaltsdefizits und Rückgewinnung (finanz-)politischer Handlungsfähigkeit, ohne soziale Spannungen und Politikverdrossenheit eskalieren zu lassen. Dass die finanziellen Einschnitte noch von der Vorgängerregierung entschieden wurden, könnte der neuen Regierung dabei entgegenkommen. Der Haushalt 2011 sieht Einsparungen von sechs Milliarden Euro vor, unter anderem bei Sozialleistungen wie Pensionen, Arbeitslosen- und Kindergeld. ${ }^{8}$ Trotz gestiegener Politikverdrossenheit wurde von Fine Gael vor der Wahl ein frischer Wind erwartet. Ihre Umfragewerte stiegen auf knapp 40 Prozent, die des traditionellen kleinen Partners, der sozialdemokratischen Labour Party, auf knapp 20 Prozent, nachdem sie zwischenzeitlich erheblich vom Protestpotential profitieren konnte. Ihr Vorsitzender, Eamon Gilmore, konnte hohe Sympatie- und Kompetenzwerte für sich verbuchen und forderte im Wahlkampf eine Verlangsamung der Sparpolitik. Auch sollen nach LabourAnsicht die Maastricht-Konvergenzkriterien erst 2016 wieder erreicht werden und nicht 2014, wie von Fianna Fáil gefordert. Die deutsche Kanzlerin Angela Merkel wurde für ihre auf europäischer Ebene geforderte Austeritätspolitik und den ,Frankfurter Weg' kritisiert. ${ }^{9}$

\section{Die Parlamentswahl 2011}

Wie im Vereinigten Königreich gibt es keinen fixen Termin für die Auflösung des Unterhauses und die Neuwahlen, sondern der Dáil muss innerhalb von fünf Jahren nach seiner ersten Sitzung aufgelöst werden (Electoral Act 1927, Sec. 7). Dies geschieht auf Vorschlag des Taoiseach durch den Staatspräsidenten. Nach der Auflösung des Dáil müssen nach der Verfassung (Art. 16.3.2) innerhalb von 30 Tagen Neuwahlen abgehalten werden. ${ }^{10} \mathrm{Wie}$ auf der Nachbarinsel ein Jahr zuvor wurden auch in Irland zum ersten Mal „Fernsehduelle“ der Parteivorsitzenden abgehalten, jeweils an den letzen vier Wochenenden vor der Wahl.

8 Vgl. Johannes Leithäuser, Zaghaft zuversichtlich, in: FAZ vom 9. Dezember 2010, S. 5.

9 Vgl. ders., a.a.O. (Fn. 6).

10 Diese Vorgabe wurde durch den Electoral Act 1992, Sec. 96, auf den 17. bis 25. Tag nach der Parlamentsauflösung präzisiert. 


\subsection{Das Wahlsystem}

Das in Irland praktizierte Wahlrecht ist eine Form der Verhältniswahl, die ansonsten lediglich in Malta für die nationalen Wahlen genutzt wird. Die "Single Transferable Vote“ (STV) ist allerdings auch als ein System eigener Art bezeichnet worden. ${ }^{11}$ Es stellt eine „listenlose Wahl nach der Proporzregel mit übertragbarer Einzelstimmgebung " 12 dar. Durch die übertragbare Einzelstimme können die Wähler alle Kandidaten auf dem Wahlzettel durchnummerieren und so ihre Präferenzen darüber zum Ausdruck bringen, in welcher Reihenfolge sie die im Wahlkreis zu vergebenden Mandate besetzt haben möchten. In den 43 Mehrpersonenwahlkreisen des Landes werden jeweils zwischen drei und fünf Abgeordnete gewählt. Insgesamt sind 166 Mandate zu vergeben, wobei der (politisch weitgehend neutralisierte) zuletzt amtierende Parlamentspräsident kompensatorisch als wiedergewählt gilt.

Den Kandidaten für einen Sitz im Dáil ist es möglich, entweder für eine Partei zu kandidieren oder als so genannter Unabhängiger anzutreten. Ein für den politischen Wettbewerb entscheidendes Merkmal ist, das es keine geschlossenen Parteilisten gibt wie etwa bei der Zweitstimme in Deutschland. Vielmehr können die Wähler ihre Präferenz direkt für einzelne Wahlkreiskandidaten angeben, die auf dem Stimmzettel alphabetisch gelistet sind. Falls der favorisierte Kandidat auf einem Stimmzettel die Quote für ein Mandat im Wahlkreis bereits erreicht hat ${ }^{13}$, werden in weiteren Auszählungsrunden die nachgeordneten Präferenzen auf den Stimmzetteln der bereits gewählten Kandidaten berücksichtigt. ${ }^{14}$ Es werden so viele Auszählungsrunden durchgeführt, bis alle Mandate eines Wahlkreises vergeben sind. Diese Regelung, die zunächst etwas kompliziert klingt, erhöht jedoch die Wirksamkeit jeder einzelnen Stimme. Im Unterschied zur relativen Mehrheitswahl in Einerwahlkreisen (first-past-the-post) gibt es deutlich weniger Stimmen, die nicht zur Wirkung kommen. Auch die Disproportionalität zwischen Stimmen- und Mandatsanteil ist geringer als im britischen Wahlsystem, allerdings höher als bei reiner Verhältniswahl. Schon ab einem landesweiten Stimmenanteil von circa 45 Prozent kann eine Partei die absolute Mehrheit der Mandate auf sich vereinigen. Das Verfahren wird von Befürwortern als besonders gerecht empfunden, da auch die Stimmen derjenigen noch zum Zuge kommen können, die mit der Erstpräferenz für bereits gewählte Kandidaten gestimmt haben.

$\mathrm{Zu}$ den kritisierten Aspekten des Wahlsystems gehört, dass der innerparteiliche Wettbewerb um die beste Vertretung lokaler Interessen in Dublin intensiviert wird. Durch den freien Wettbewerb um Stimmen zwischen allen Kandidaten auf einem Stimmzettel müssen nicht nur einfache Abgeordnete, sondern selbst Minister sich gegen Kandidaten auch aus der eigenen Partei durchsetzen. Dies gilt insbesondere für Fianna Fáil, bei der zwischen 1927 und 199756 Prozent nicht wiedergewählter Abgeordneter durch Konkurrenten aus der eigenen Partei ersetzt wurden. ${ }^{15}$ Dadurch findet eine starke Bindung von Zeit und In-

11 Vgl. Dieter Nohlen, a.a.O. (Fn. 3), S. 338.

12 Ebenda.

13 Bei der Auszählung wird zunächst eine Droop-Quota gebildet, die sich über die Formel Gesamtzahl der gültigen Stimmen dividiert durch die mit eins addierte Anzahl der Sitze pro Wahlkreis plus eins berechnet.

14 Vgl. Jürgen Elvert, Das politische System Irlands, in: Wolfgang Ismayr (Hrsg.), Die politischen Systeme Westeuropas, Wiesbaden 2009, S. 307 - 348, S. 320.

15 Vgl. Trinity College Dublin, Ireland's PR-STV Electoral System: A Need for Reform?, Dublin 2010, http://www.tcd.ie/Political_Science/staff/michael_gallagher/IrishElectSys.php (Abruf am 3. März 2011). 
teressen der Abgeordneten an lokale Politik statt. Zeitweise hatten über 90 Prozent von ihnen auch kommunale Mandate inne, denn ohne diese gibt es offenkundig erhebliche Probleme, in den Dáil gewählt zu werden. Bei der Formulierung nationaler Politiken haben deswegen oft Beamte und politische Berater großen Einfluss.

Die Wahlkreiseinteilung unterliegt einer kontinuierlichen Veränderung durch die Politik. Bis 1980 ist es immer wieder zur Verringerung der Mandatszahl in den Wahlkreisen gekommen. ${ }^{16}$ Damals wurde eine unabhängige Wahlkreiskommission gebildet, die parteipolitische Willkür („gerrymandering“) bei der Zuschneidung der Wahlkreise drastisch gesenkt hat. Nach der Verfassung darf die Mandatszahl nicht unter drei sinken. Von den 43 Wahlkreisen entsenden 15 (35 Prozent) vier Abgeordnete, elf (25 Prozent) fünf und 17 (40 Prozent) drei. Durch diesen hohen Anteil von Dreipersonenwahlkreisen wird die höhere Proportionalität größerer Mehrpersonenwahlkreise kaum genutzt. Aber auch in Dreierwahlkreisen kann ein Kandidat schon mit etwa elf Prozent der Erstpräferenzen Erfolg haben. Dadurch haben auch parteiunabhängige Kandidaten gute Chancen. „Tendenziell werden dadurch stimmstarke Parteien auf Kosten stimmschwacher (ohne geographisch konzentrierte Wählerschaft) bevorteilt. "17 Auch wenn die Berücksichtigung niedrigerer Präferenzen den Disproportionalitätseffekt gegenüber der Mehrheitswahl abschwächt, so bleibt doch immer ein beachtlicher Anteil von Stimmen, die nicht in Mandate übertragen werden und zu einer Überrepräsentation der beiden stimmstärksten Parteien führen. ${ }^{18}$ Auch bei der Auswirkung auf das Regierungsformat bewegt sich das irische Wahlsystem zwischen Mehrheits- und Verhältniswahl. Die parlamentarische Mehrheitsbildung durch eine einzelne Partei wird zwar erleichtert, aber doch nicht zur Regel. Allein Fianna Fáil gelang es einige Male, absolute Parlamentsmehrheiten zu erzielen. Häufiger sind Kleine Koalitionen, aber auch Minderheitsregierungen sind keine Seltenheit. Wie beim Mischsystem in Deutschland ist in Irland strategisches Wählen möglich. Dabei spielt es eine erhebliche Rolle, ob die Unterstützer einer potenziellen Koalition ihre Präferenzen untereinander aufteilen oder nicht.

\subsection{Das Wahlergebnis 2011}

Die Wahlbeteiligung reichte 2011 mit über 70 Prozent wieder an das Niveau der 1980er Jahre heran. Das Wahlergebnis kam nach den Umfragen, die Fine Gael teilweise bis zu 40 Prozent der Erstpräferenzen voraussagten, wenig überraschend. Die tatsächlich erzielten 36,23 Prozent sind das beste Ergebnis der Partei seit 1982, allerdings reichte es trotz der beachtlichen Disproportionalität eines um 9,55 Prozent höheren Sitzanteils nicht für eine absolute Mehrheit. Als Koalitionspartner sprang die Labour Party ein, die mit 19,35 Prozent der Stimmen und 22,3 Prozent der Sitze ihr bisher bestes Ergebnis erzielte und damit zum kleinen Partner einer Großen Koalition avancierte. Dagegen erzielte die strukturelle Mehrheitspartei Fianna Fáil mit 17,36 Prozent der Stimmen und zwölf Prozent der Sitze das mit Abstand schlechteste Ergebnis ihrer Geschichte. Gegenüber 2007 sank ihr Stimmenanteil um 58,23 Prozent, ihr Mandatsanteil um 74,36 Prozent.

16 Vgl. Dieter Nohlen, a.a.O. (Fn. 3), S. 340.

17 Ebenda, S. 341.

18 Vgl. ebenda, S. 342. 


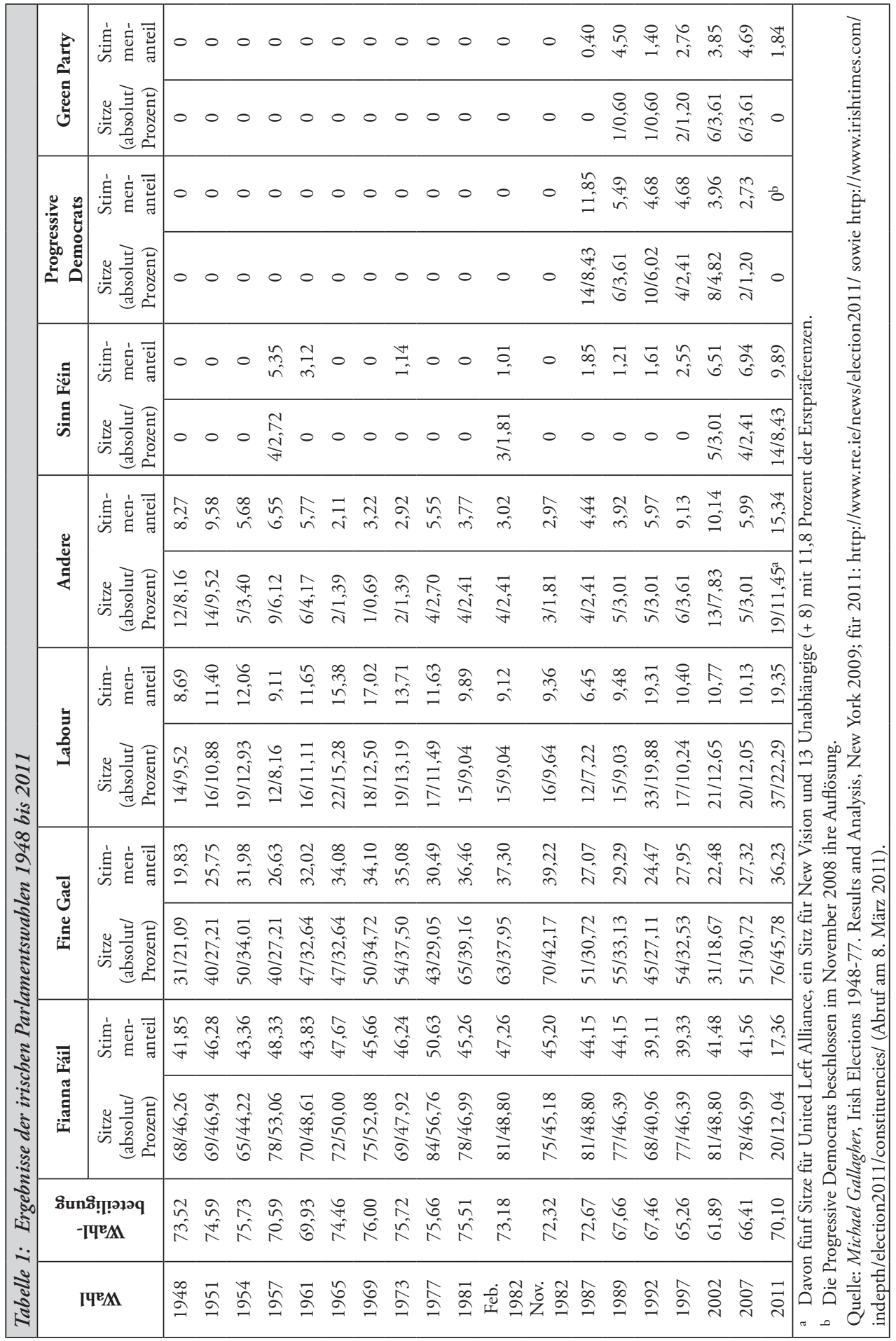




\begin{tabular}{|l|c|c|c|c|c|}
\hline \multicolumn{7}{|c|}{ Tabelle 2: Strukturmerkmale des irischen Parteiensystems } \\
\hline \multicolumn{2}{|c|}{ Wahl } & $\begin{array}{c}\text { Sitzanteil } \\
\text { FF und FG } \\
\text { (absolut / in Prozent) }\end{array}$ & $\begin{array}{c}\text { Stimmenanteil } \\
\text { FF und FG } \\
\text { (in Prozent) }\end{array}$ & $\begin{array}{c}\text { Effektive Zahl } \\
\text { parlamentarischer } \\
\text { Parteien }\end{array}$ & $\begin{array}{c}\text { Disproportionalität } \\
\text { (Gallagher-Index) }\end{array}$ \\
\hline 1948 & 99 & 67,35 & 61,68 & 3,66 & 5,59 \\
1951 & 109 & 74,15 & 72,03 & 3,26 & 2,60 \\
1954 & 115 & 78,23 & 75,34 & 3,01 & 2,35 \\
1957 & 118 & 80,27 & 74,96 & 2,72 & 4,40 \\
1961 & 117 & 81,25 & 75,85 & 2,78 & 4,46 \\
1965 & 119 & 82,64 & 81,75 & 2,61 & 2,17 \\
1969 & 125 & 86,81 & 79,76 & 2,46 & 5,38 \\
1973 & 123 & 85,42 & 81,32 & 2,59 & 2,40 \\
1977 & 127 & 85,81 & 81,12 & 2,36 & 4,91 \\
1981 & 143 & 86,14 & 81,72 & 2,62 & 2,73 \\
Feb. 1982 & 144 & 86,75 & 84,56 & 2,53 & 1,69 \\
Nov. 1982 & 145 & 87,35 & 84,42 & 2,52 & 2,74 \\
1987 & 132 & 79,52 & 71,22 & 2,89 & 5,14 \\
1989 & 132 & 79,52 & 73,44 & 2,94 & 3,85 \\
1992 & 113 & 68,07 & 63,58 & 3,46 & 3,10 \\
1997 & 131 & 78,92 & 67,28 & 3,00 & 6,55 \\
2002 & 112 & 67,47 & 63,96 & 3,38 & 6,62 \\
2007 & 129 & 77,71 & 68,88 & 3,03 & 5,85 \\
2011 & 96 & 56,83 & 57,30 & 3,50 & 8,35 \\
\hline Quelle: Michael Gallagher, Irish Elections & $1948-77$. Results and Analysis, New York 2009; für 2011: \\
http://www.rte.ie/news/election2011/ sowie http://www.irishtimes.com/indepth/election2011/constitu- \\
encies/ (Abruf am 8. März 2011). Eigene Berechnung. & & \\
\hline
\end{tabular}

Um das zweieinhalbfache gesteigert gegenüber 2007 hat sich die Unterstützung für die Unabhängigen und Kleinparteien, die nun mit insgesamt 19 Mandaten (11,45 Prozent) im Dáil vertreten sind. ${ }^{19}$ Sinn Féin, die einzige gesamtirische Partei, konnte mit 9,89 Prozent ihr bestes Ergebnis erzielen. Ihren Mandatsanteil konnte sie verdreieinhalbfachen. Mit 8,43 Prozent der Sitze ist die Disproportionalität von allen Parteien bei Sinn Féin diesmal am schwächsten ausgeprägt. Gerry Adams, der von Nordirland in die Republik wechselte, konnte im grenznahen Wahlkreis Loth mit 21,7 Prozent das beste Resultat für Sinn Féin verbuchen. Der Stimmenanteil der Grünen brach um über die Hälfte ein, sie verloren alle Sitze, einschließlich der ihrer beiden ehemaligen Minister. Die Partei wurde für die Fehler ihres Koalitionspartners mit abgestraft und konnte sich, anders als Sinn Féin, auch nicht als Protestpartei profilieren. Aufschlussreich ist auch, dass der Zusammenschluss linker Kleinparteien „United Left Alliance“ (insgesamt fünf Sitze) nicht stärker von der massiven Wirtschaftskrise profitieren konnte.

Der kumulierte Anteil der Stimmen der beiden bis 2007 größten Parteien, Fianna Fáil und Fine Gael, ist auf 57,3 Prozent und damit auf das niedrigste Niveau seit dem Zweiten

19 „Some independent MPs in the outgoing parliament were known as ,gombeens - meaning unprincipled wheeler-dealers. But the financial crisis has thrown up a different breed in this election."John Murray Brown, Poll transforms Irish political landscape, in: Financial Times vom 27. Februar 2011. 
Weltkrieg gefallen, nachdem er 1982 mit 84,56 Prozent einen Höchststand erreicht hatte. Ähnlich sieht es mit dem kumulierten Anteil der Sitze der beiden größten Parteien aus, der in der Novemberwahl 1982 am größten ausfiel.

Das markanteste Merkmal der Wahl ist die hohe Volatilität. Nach dem Pedersen-Index, also der Hälfte der Summe von Gewinnen und Verlusten der Parteien, errechnet sich eine Netto-Volatilität von 29,6 Prozent. Dies ist der dritthöchste Wert in Europa seit 1945, nach Italien 1994 und den Niederlanden 2002. In beiden Fällen entstand die hohe Volatilität aber insbesondere durch den Aufstieg neuer Parteien (Forza Italia und Liste Pim Fortuyn). ${ }^{20}$ In Irland ist die Volatilität 2011 dagegen nicht mit einer neuen Partei verbunden gewesen. Ein weiteres Strukturmerkmal des irischen Parteiensystems ist die effektive Zahl der parlamentarischen Parteien: Sie bewegt sich seit den 1990er Jahren bei über drei. 2011 ist mit 3,5 der dritthöchste Wert seit 1948 erreicht, der aber immer noch im europäischen Mittelfeld liegt. Neben dem Ausmaß der Fragmentierung ist die Polarisierung ein wichtiges Merkmal eines Parteiensystems. Die ideologische Spannweite ist in Irland traditionell niedrig und hat seit den 1970er Jahren immer wieder Anlass zu Kommentaren über die kaum unterscheidbaren Profile der Parteien gegeben. ${ }^{21}$ Seit Ende der 1980er Jahre verstärkt Koalitionen notwendig wurden, hat sich die Priorität von „office, votes, and then policy“ weiter verstärkt. Mit den Niederlanden, Österreich, Spanien und besonders Portugal gehörte Irland in den 1990er Jahren zu den am wenigsten polarisierten Parteiensystemen. ${ }^{22}$ Mit den Grünen zog zwischenzeitlich eine stärker polarisierende Partei ins Parlament, die aber 2011 den Einzug in den Dáil verpasste; und Labour dürfte durch die Regierungsbeteiligung noch weniger Spielraum für Polarisierung haben als zuvor in der Opposition.

Die durch die STV ausgelöste Disproportionalität, das heißt die Abweichung des Sitzanteils vom Stimmenanteil, ist eher moderat. Nach dem Gallagher-Index kommt Arend Lijphart auf einen Durchschnittswert von 8,25 Prozent der 36 untersuchten Staaten zwischen 1945 und 1996, wobei die Niederlande mit 1,3 Prozent den niedrigsten Wert aufweisen und Frankreich mit 21 Prozent den höchsten. ${ }^{23}$ Irland liegt mit 3,45 Prozent an dreizehnter Stelle deutlich unter dem Durchschnitt, wobei allerdings nur die Erstpräferenzen berücksichtigt werden. Neben dem Gallagher-Index gibt es eine Reihe weiterer Möglichkeiten, Disproportionalität zu erfassen. So kann statt eines Indexwertes für die Disproportionalität eines ganzen Wahlsystems auch die einzelner Parteien(gruppen) näher betrachtet werden, da diese für einzelne Parteien aufgrund von Wahlkreisgröße und -zuschnitt sowie geographischer Verteilung der politischen Unterstützung unterschiedlich ausfallen kann.

20 Vgl. Peter Mair, One of Europe's Most Volatile Elections, http://politicalreform.ie/2011/02/28/ one-of-europe's-most-volatile-elections/ (Abruf am 26. März 2011).

21 Vgl. Maura Adshead/ Jonathan Tonge, a.a.O. (Fn. 1), S. 87.

22 Vgl. Thomas Saalfeld, a.a.O. (Fn. 2), S. 191. Dies kann auf die weitgehende Abwesenheit von Klassengegensätzen, konfessionellen (beziehungsweise laizistischen) Unterschieden und StadtLand-Unterschieden zurück geführt werden. Lediglich das Zentrum-Peripherie-Cleavage konnte eine größere Prägekraft entfalten, vgl. Maura Adshead / Jonathan Tonge, a.a.O. (Fn. 1), S. 90.

23 Vgl. Arend Lijphart, Pattern of Democracy, New Haven 1999, S. 162; Michael Gallagher, Proportionality, Disproportionality and Electoral Systems, in: Electoral Studies, 10. Jg. (1991), H. 1, S. 33 - 51. Der Gallagher-Index berechnet sich nach der Formel $G=\sqrt{ } 1 / 2 \sum$ (vi - si) ${ }^{2}$, wobei vi der prozentuale Stimmenanteil, si der prozentuale Mandatsanteil einer Partei ist. Der aggregierte Index erfasst die gesamte von einem Wahlsystem ausgehende Disporportionalität. 
Dies trifft insbesondere für die Varianten des Mehrheitswahlrechts $\mathrm{zu}^{24}$, jedoch produziert auch die Verhältniswahl mit ihren Gestaltungselementen deutliche Abweichungen von perfekter Proportionalität. Auch Verhältniswahlsysteme wie die STV, in denen das technische „Auffangen“ ansonsten verlorener Stimmen ein hohes Gewicht hat, zeigen für unterschiedlich große Parteien unterschiedliche Responsivität. ${ }^{25}$

Auf empirischer Ebene werden Stimmenzugewinne (-verluste) einzelner Parteien in unterschiedlichem Maß in Sitzzugewinne (-verluste) übersetzt. Dabei kann es zum Beispiel zu unterschiedlichen Disproportionalitäten kleiner und großer Parteien kommen. ${ }^{26}$ Cox / Shugart halten insbesondere die politische Dimension von Disproportionalität für untersuchenswert, speziell die Frage, ob und welche Parteien aufgrund ihrer Größe unter- und überrepräsentiert werden: „We suggest that less attention be paid to disproportionality and more attention paid to the extent to which different methods of PR favour large parties over small. This, after all, has the more direct impact on the party system." 27 Um den Einfluss eines Wahlsystems auf die Disproportionalität einzelner Parteien und damit einen möglichen „big party bias“ abschätzen zu können, können verschiedene Verfahren angewendet werden, zum Beispiel die Untersuchung der Häufigkeit von „manufactured majorities" 28 oder des Anteils von Stimmen, unterhalb dessen die Parteien tendenziell unterrepräsentiert werden. ${ }^{29}$ Eine weitere Möglichkeit besteht darin, das Verhältnis von Stimmen- und Sitzanteilen für die einzelnen Parteien in einer Wahl graphisch aufzubereiten. ${ }^{30}$

Für die Wahl 2011 zeigt sich bei dieser Vorgehensweise, dass die beiden erfolgreichsten Parteien (FG und Labour) ihren Stimmenanteil überproportional in Mandate umsetzen konnten, während die „Kleinparteien“ Fianna Fáil und Sinn Féin sowie die unabhängigen Kandidaten ihre Stimmen nur unterproportional in Mandate transformieren konnten. Abbildung 1 legt nahe, dass ab einem Stimmanteil von knapp 20 Prozent ein Wechsel von unter- zu überproportionaler Transformation stattfindet. Abbildung 2 mit den Ergebnissen aller seit $1981^{31}$ mandatswirksamen Parteien senkt die Schwelle des Übergangs von unterzu überproportionaler Repräsentation noch etwas, bestätigt aber die Tendenz. Sowohl der Gallagher-Index (vgl. Tabelle 2) als auch eine einfache lineare Regression der Stimmen- und Sitzanteile 2011 (siehe Abbildung 1) im Vergleich zum Durchschnitt der letzten 30 Jahre (siehe Abbildung 2) zeigt die deutlich gestiegene Disproportionalität im irischen Parteiensystem bei der letzten Wahl.

Die durchgezogene Linie ist eine einfache lineare Regressionsgrade (ols) von Sitz- auf Stimmenanteilen. Die nicht-standardisierte Steigung dieser Linie gibt nach Cox / Shugart

24 Vgl. Philip Manow, Dimensionen der Disproportionalität - Erststimmen und Direktmandate in den Bundestagswahlen von 1953 bis 2009, in: PVS, 51. Jg. (2010), H. 3, S. 433 - 455.

25 Vgl. ebenda.

26 Vgl. Gary W. Cox / Matthew S. Shugart, Comment on Gallagher's 'Proportionality, Disproportionality and Electoral Systems', in: Electoral Studies, 10. Jg. (1991), H. 4, S. 348 - 352.

27 Ebenda, S. 350.

28 Douglas W. Rae, The Political Consequences of Electoral Laws, New Haven 1967, S. 74.

29 Vgl. Rein Taagepera / Markku Laakso, Proportionality Profiles of West European Electoral Systems, European Journal of Political Research, 8. Jg. (1980), H. 4, S. $423-446$.

30 Vgl. Gary W. Cox / Matthew S. Shugart, a.a.O. (Fn. 26), S. 350.

311981 nahm eine unabhängige Wahlkreiskommission ihre Arbeit auf, die der Praxis der Revision von Wahlkreisgrenzen durch das Parlament, das heißt der Regierungsmehrheit, ein Ende bereitete; vgl. Michael Gallagher / Liam Weeks, Ireland, in: Dieter Nohlen / Philipp Stöver (Hrsg.), Elections in Europe, Baden-Baden, S. 987 - 1025, S. 990. 


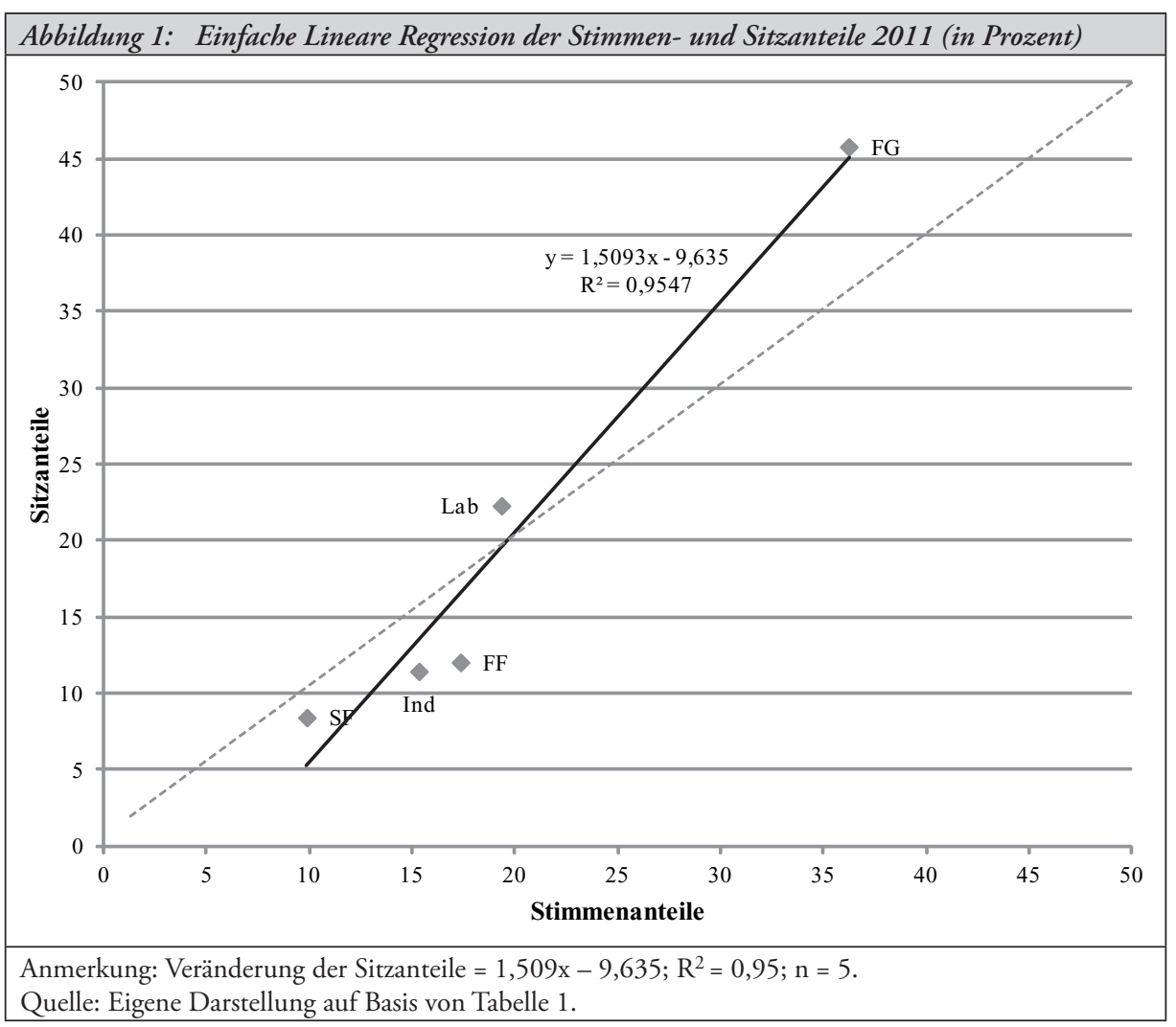

einen einfachen Index des „big-party bias“ eines Wahlsystems in einem Land an. Mit einem Wert von 1,5 zeigt sich 2011 eine im Vergleich zum Durschnitt der letzten 30 Jahre (siehe Abbildung 2) hohe Disproportionalität zugunsten großer Parteien. Jeder Punkt in der Graphik zeigt den Stimmenanteil (x-Achse) und den Mandatsanteil (y-Achse) für eine irische Partei bei einer nationalen Wahl zwischen 1981 und 2011 an. Bei einem Wert unter 1 wären die größeren Parteien im Vergleich zu ihrem Stimmenanteil im Parlament unterrepräsentiert und die kleineren überrepräsentiert. Bei einem Wert von exakt eins (perfekte Proportionalität) sind kleine und große Parteien gleichermaßen proportional vertreten (siehe unterbrochene Linie in den Abbildungen). 32

Betrachtet man die Disproportionalität in den Wahlen von 1981 bis 2011 getrennt, fällt die unterschiedliche Steigung der Regressionsgrade der einzelnen Parteien auf. Wie Abbildung 3 zeigt, ist die Proportionalität von Stimmen- und Mandatsanteilen bei Labour mit $1,13\left(R^{2}=0,95\right)$ am größten. Trotz des aktuellen Stimmeneinbruchs von Fianna Fáil ist bei ihr die „Bevorzugung“ durch das Wahlsystem im Längsschnitt der letzten 30 Jahre immer noch am größten. 


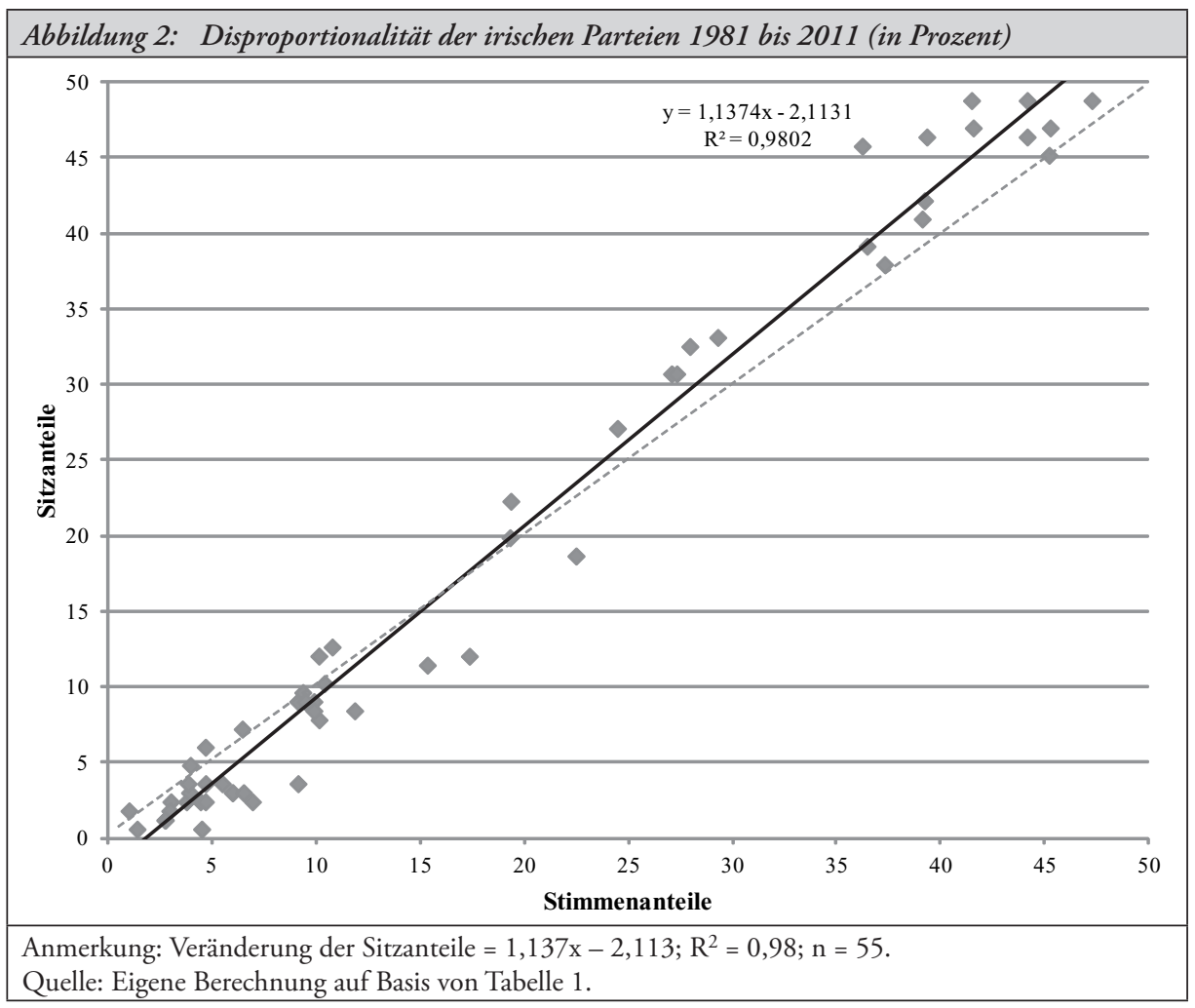

Für eine absolute Mehrheit im Dáil sind 83 Sitze erforderlich. ${ }^{33}$ Fine Gael (76) und Labour Party (37) kommen zusammen auf 113 Sitze (68 Prozent). In der Logik der formalen Koalitionstheorie ist dies für die stärkste Partei eine denkbar ungünstige Konstellation, denn es erhöht das Erpressungspotential des kleinen Koalitionspartners. Um eine „minimum winning coalition" zu bilden und die Einflusschancen in der Regierung zu maximieren, wäre in der formalen Logik der Koalitionstheorie eine von mindestens acht Unabhängigen gestützte Minderheitsregierung die beste Lösung gewesen, gefolgt von einer Koalition mit Sinn Féin (14) oder mit Fianna Fáil (20). Die von Enda Kenny präferierte Koalition mit Labour wurde damit begründet, dass Irland eine stabile und starke Regierung brauche. ${ }^{34}$ Obwohl sich beide Parteien in Wahlkampf heftig attackiert hatten, konnten sie sich nach der Wahl schnell auf ein Regierungsprogramm einigen. Aufgrund der Schwäche von Fianna Fáil stellt die Formation nun aber zum ersten Mal in der irischen Geschichte eine Große Koalition der beiden stärksten Parteien dar. Von einer solchen Konstellation sind allerdings weniger strukturelle Reformen als vielmehr eine Verwässerung der noch von der Fianna Fáil-Regierung mit EU und IWF ausgehandelten Bedingungen des Rettungspaketes zu erwarten.

33 Das 166. Mitglied, der Ceann Comhairle des Dáil, ist den parteipolitischen Kontroversen weitgehend entzogen. Er kann (und muss) seine Stimme gemäß Artikel 15,11 $2 f$ der Verfassung lediglich bei Stimmengleichheit ausüben.

34 Vgl. John Murray Brown, a.a.O. (Fn. 19). 


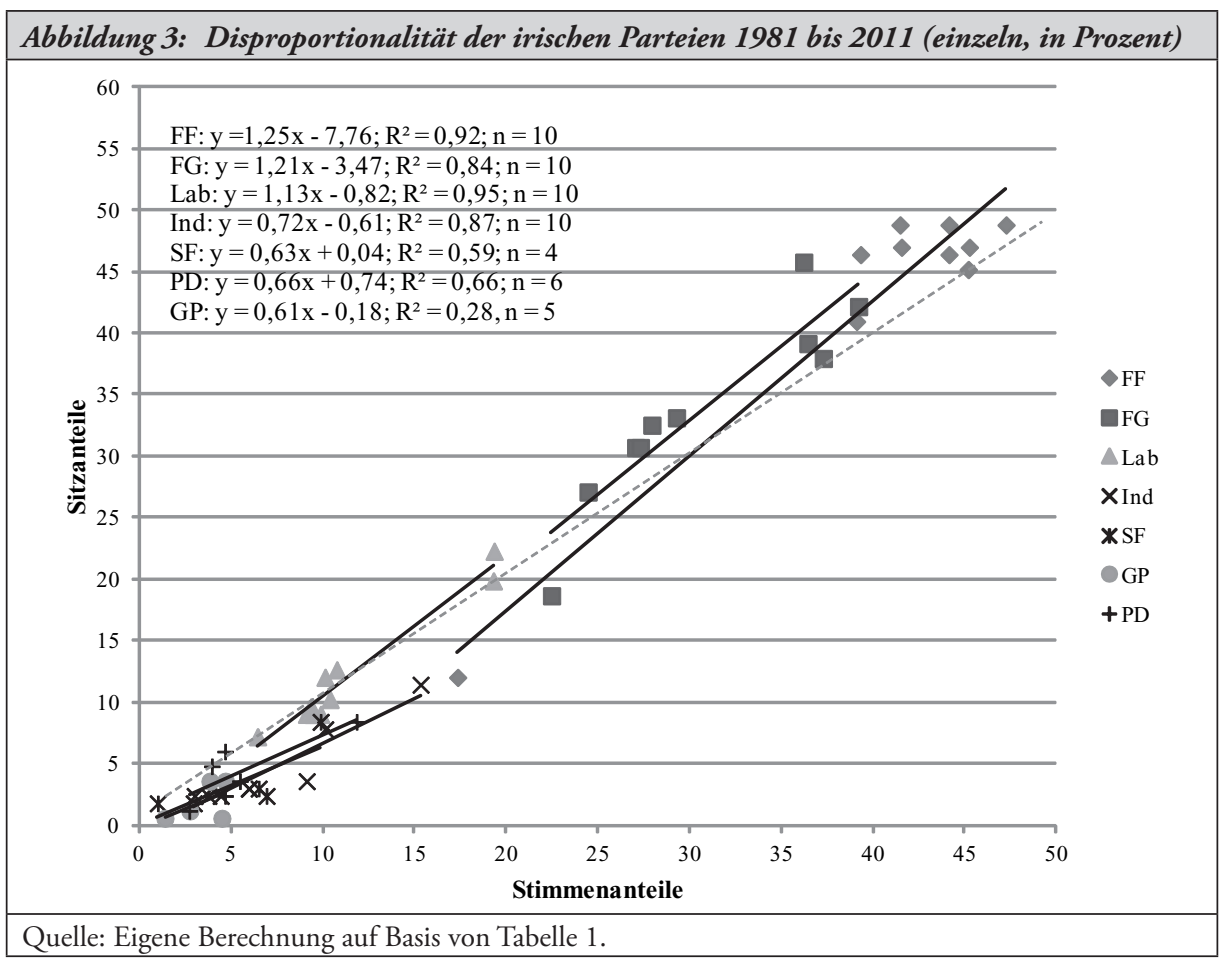

\section{Die Regierungsbildung}

Während Fine Gael die Koalitionsvereinbarung lediglich durch ein Treffen der Parlamentsfraktion absegnen ließ, musste Labour nach Parteistatut eine Delegiertenversammlung mit circa 1.000 Mitgliedern abhalten. Unbehagen wurde insbesondere vom linken Flügel geäußert. Allerdings befanden sich die beiden neuen Regierungsparteien zuvor 14 Jahre in der Opposition. Ein Labour-Politiker interpretierte die Veränderung des Parteiensystems als eine Annäherung an kontinentaleuropäische Cleavages: „In line with the European norm, our largest party is now Christian Democratic and our second-largest party is Social Democratic. Civil war politics is now finished." 35 Wenn auch überzeichnet, zeigt dies doch eine wahrgenommene Entwicklungstendenz an.

Von der Wahl am 25. Februar bis zur Bekanntgabe der Koalitionsvereinbarung am 6. März vergingen gerade einmal neun Tage, von denen lediglich die letzten fünf für die Aushandlung des Regierungsprogramms benötigt wurden. Beide Parteiführungen wurden dabei umfangreich von Fachleuten beraten. „The support groups provided financial, economic, legal advice and research functions to the teams, as well as writing position papers and being involved in producing drafts of the programme for government."36 Der Titel des Koalitions-

35 Ebenda.

36 Harry $M c G e e$, Party experts played central role in shaping programme for government, in: The Irish Times vom 8. März 2011, http://www.irishtimes.com/newspaper/ireland/2011/0308/ 1224291590521.html (26. März 2011). 
vertrages „Government for National Recovery” und Selbstbezeichnungen wie „national government” sind auch eine Anspielung auf die "nationale Koalition” von Fine Gael und Labour von 1973 unter Liam Cosgrave, als Fine Gael nach 16 Oppositionsjahren eine erfolgreiche Koalitionsregierung formen konnte - die 1977 aber zu einer bitteren Wahlniederlage für die Partei führte. ${ }^{37}$ Auch 1987 endete eine weitere Regierungskoalition (unter Garret FitzGerald) von Fine Gael und Labour nach einer Wahlperiode in einer schweren Niederlage für Fine Gael. Erst 1997 änderte sich dieses Muster, als sich die Partei nach drei Jahren „Regenbogenkoalition" (unter Taoiseach John Bruton) mit Labour und Democratic Left erstmals nach einer Regierungsperiode verbessern konnte. Allerdings waren seinerzeit die Stimmenverluste von Labour zu drastisch, um das Bündnis fortführen zu können.

In der Koalitionsvereinbarung 2011 wurde unter anderem verabredet, eine mögliche Abschaffung der Zweiten Kammer (Seanad) in einem Referendum zur Diskussion zu stellen. Außerdem soll ein Verfassungskonvent einberufen werden, um über verschiedene Ergänzungen der Verfassung zu beraten, unter anderem eine Verkürzung der Amtszeit des Präsidenten von sieben auf fünf Jahre. $\mathrm{Zu}$ den vorgeschlagenen Reformen im Bereich legislativer Verfahren gehören eine Ausweitung der Fragestunden sowie eine Begrenzung der Anzahl der Ausschüsse, wobei die wichtigsten in der Verfassung verankert werden sollen. Des Weiteren wurde eine Begrenzung von Wahlkampfausgaben, die Prüfung einer Senkung des Wahlalters auf 17 und die Verbesserung der Repräsentation von Frauen vereinbart. Zu den tagespolitischen Forderungen des „Programme for national Government” gehört eine Stärkung des Wirtschaftsministeriums, das mit der Nachverhandlung des EU/IWF-Rettungspakets beauftragt werden soll. Die Kontrolle der Ausgaben und des öffentlichen Sektors soll vom Finanzministerium in das Ministerium für Public Service Reform verlagert werden.

Innerhalb des Kabinetts wird ein Wirtschaftsrat aus vier Ministerien mit primärer Verantwortung für die Bewältigung der Wirtschaftskrise eingerichtet. Von der Labour Party, die sich besonders für die Nachverhandlung mit EU und IWF stark gemacht hat, wurde auch die Gründung einer strategischen Investitionsbank eingebracht, die Unternehmen helfen soll, die sich von den „high street banks" abgewendet haben. Von den von Fine Gael ursprünglich geforderten 30.000 Stellenstreichungen im öffentlichen Dienst bis 2014 sind 18.000 bis 21.000 freiwillige Abfindungen bis 2013 und die anschließende Prüfung weiterer 5000 Stellen im Koalitionsvertrag geblieben..$^{38}$

Am 9. März wurde Kenny mit 117 gegen 27 Stimmen zum neuen Premierminister gewählt. ${ }^{39}$ Die fünf zusätzlichen Stimmen (zu den 113 eigenen der Koalition) kommen vermutlich aus dem Kreis der Unabhängigen. Das neue Kabinett besteht aus zehn FG- und fünf Labour-Ministern, was in etwa den Anteilen der die Regierung tragenden Fraktionen entspricht. Überraschenderweise erhielt die Labour-Fraktionsvorsitzende Joan Burton nicht das Ministerium für Public Sector Reform oder Finanzen, was allgemein erwartet wurde, sondern Social Protection. Nach der Bekanntgabe der Kabinettsverteilung wurden weitere

37 Auch das im Oktober 1987 vom National Economic and Social Council (NESC) beschlossene „Programme for National Recovery“, eine Art korporatistischer Drei-Jahres-Plan, klingt hierbei an; vgl. Jürgen Elvert, a.a.O. (Fn. 14), S. 327.

38 Vgl. Fine Gael, Labour agree to form coalition government, in: The Irish Times vom 7. März 2011, http://www.irishtimes.com/newspaper/breaking/2011/0306/breaking23.html (Abruf am 26. März 2011).

39 Nach der Wahl im Dail geht der neue Taoiseach zum Áras an Uachtaráin, dem Amtssitz des Präsidenten (aktuell Mary McAleese), um seine Dienstsiegel im Empfang zu nehmen. 
15 Juniorminister (Staatssekretäre) ernannt, neun aus den Reihen der Fine Gael-Abgeordneten, sechs von Labour, wodurch diese hier ein leichtes Übergewicht bekam. Dabei wurden auch einige Abgeordnete berücksichtigt, die zuvor als Kandidaten für ein Ministeramt gehandelt worden, aber nicht zum Zuge gekommen waren. Juniorministern ist ein spezieller Aufgabenbereich innerhalb eines oder zweier Ressorts zugeordnet. Insgesamt haben sechs Juniorminister Aufgaben in zwei verschiedenen Ministerien übernommen. Zehnmal wurde bei der Vergabe dieser Positionen das Kreuzstichverfahren angewendet, das heißt

\begin{tabular}{|c|c|c|c|}
\hline \multicolumn{4}{|c|}{ Tabelle 3: Kabinett Enda Kenny (Stand: März 2011) } \\
\hline Ministerium & Seniorminister & Juniorminister & Aufgabenbereich JM \\
\hline Taoiseach & $\begin{array}{l}\text { Enda Kenny } \\
\text { (FG) }\end{array}$ & $\begin{array}{l}\text { Paul Kehoe* }(\mathrm{FG}) \\
\text { Lucinda Creighton }(\mathrm{FG})\end{array}$ & $\begin{array}{l}\text { Government Chief Whip } \\
\text { European Affairs }\end{array}$ \\
\hline $\begin{array}{l}\text { Agriculture, Marine } \\
\text { and Food }\end{array}$ & $\begin{array}{l}\text { Simon Coveney } \\
\text { (FG) }\end{array}$ & Shane McEntee (FG) & $\begin{array}{l}\text { Food, Horticulture and } \\
\text { Food Safety }\end{array}$ \\
\hline $\begin{array}{l}\text { Arts, Heritage and } \\
\text { Gaeltacht Affairs }\end{array}$ & $\begin{array}{l}\text { Jimmy Deenihan } \\
(\mathrm{FG})\end{array}$ & Dinny McGinley (FG) & Gaeltacht Affairs \\
\hline Children & $\begin{array}{l}\text { Frances Fitzgerald } \\
\text { (FG) }\end{array}$ & & \\
\hline $\begin{array}{l}\text { Communications, Energy } \\
\text { and Natural Resources }\end{array}$ & $\begin{array}{l}\text { Pat Rabbitte } \\
\text { (Lab) }\end{array}$ & Fergus O’Dowd (FG) & New Era Project \\
\hline Education and Skills & $\begin{array}{l}\text { Ruairi Quinn } \\
\text { (Lab) }\end{array}$ & $\begin{array}{l}\text { Ciaran Cannon }(\mathrm{FG}) \\
\text { Seán Sherlock }(\mathrm{Lab})\end{array}$ & $\begin{array}{l}\text { Training and Skills Re- } \\
\text { search and Innovation }\end{array}$ \\
\hline $\begin{array}{l}\text { Enterprise, Jobs and } \\
\text { Innovation }\end{array}$ & $\begin{array}{l}\text { Richard Bruton } \\
\text { (FG) }\end{array}$ & $\begin{array}{l}\text { John Perry (FG) } \\
\text { Seán Sherlock (Lab) }\end{array}$ & $\begin{array}{l}\text { Small Business } \\
\text { Research and Innovation }\end{array}$ \\
\hline $\begin{array}{l}\text { Environment, Community } \\
\text { and Local Government }\end{array}$ & $\begin{array}{l}\text { Phil Hogan } \\
\text { (FG) }\end{array}$ & $\begin{array}{l}\text { Willie Penrose* (Lab) } \\
\text { Fergus O'Dowd (FG) }\end{array}$ & $\begin{array}{l}\text { Housing and Planning } \\
\text { New Era Project }\end{array}$ \\
\hline Finance & $\begin{array}{l}\text { Michael Noonan } \\
\text { (FG) }\end{array}$ & Brian Hayes (FG) & $\begin{array}{l}\text { Public Service Reform and } \\
\text { OPW }\end{array}$ \\
\hline $\begin{array}{l}\text { Foreign Affairs } \\
\text { and Trade }\end{array}$ & $\begin{array}{l}\text { Eamon Gilmore } \\
\text { (Lab), zugl. } \\
\text { Tánaiste }\end{array}$ & $\begin{array}{l}\text { Jan O’Sullivan (Lab) } \\
\text { Lucinda Creighton (FG) }\end{array}$ & $\begin{array}{l}\text { Trade and Development } \\
\text { European Affairs }\end{array}$ \\
\hline Health & $\begin{array}{l}\text { James Reilly } \\
\text { (FG) }\end{array}$ & $\begin{array}{l}\text { Róisin Shortall (Lab) } \\
\text { Kathleen Lynch (Lab) }\end{array}$ & $\begin{array}{l}\text { Primary Care } \\
\text { Disability, Equality and } \\
\text { Mental Health }\end{array}$ \\
\hline $\begin{array}{l}\text { Justice, Equality } \\
\text { and Defence }\end{array}$ & $\begin{array}{l}\text { Alan Shatter } \\
\text { (FG) }\end{array}$ & $\begin{array}{l}\text { Kathleen Lynch (Lab) } \\
\text { Paul Kehoe (FG) }\end{array}$ & $\begin{array}{l}\text { Disability, Equality and } \\
\text { Mental Health Defence }\end{array}$ \\
\hline $\begin{array}{l}\text { Public Expenditure } \\
\text { and Reform }\end{array}$ & $\begin{array}{l}\text { Brendan Howlin } \\
\text { (Lab) }\end{array}$ & Brian Hayes (FG) & $\begin{array}{l}\text { Public Service Reform and } \\
\text { the Office of Public Works } \\
\text { (OPW) }\end{array}$ \\
\hline Social Protection & $\begin{array}{l}\text { Joan Burton } \\
\text { (Lab) }\end{array}$ & & \\
\hline $\begin{array}{l}\text { Transport, Tourism } \\
\text { and Sport }\end{array}$ & $\begin{array}{l}\text { Leo Varadkar } \\
\text { (FG) }\end{array}$ & $\begin{array}{l}\text { Michael Ring (FG) } \\
\text { Alan Kelly (Lab) }\end{array}$ & $\begin{array}{l}\text { Tourism and Sport } \\
\text { Public and Commuter } \\
\text { Transport }\end{array}$ \\
\hline \multicolumn{4}{|c|}{$\begin{array}{l}\text { * Mit Kabinettsrang. } \\
\text { Quelle: Stephen Collins, a.a.O. (Fn. 41); Steven Carroll, } 15 \text { Junior ministers Appointed, in: The Irish } \\
\text { Times vom 10. März 2011, http://www.irishtimes.com/newspaper/breaking/2011/0310/breaking47. } \\
\text { html (Abruf am 11. April 2011). }\end{array}$} \\
\hline
\end{tabular}


diese Amsinhaber gehören einer anderen Partei an als der Seniorminister ihres Hauses. ${ }^{40} \mathrm{Je}$ ein von Fine Gael (Children) und Labour (Social Protection) geführtes Ressort hat keine Juniorminister. Als neue Generalstaatsanwältin und erste Frau in diesem Amt wurde die bisherige Labour-Schatzmeisterin Máire Whelan ernannt. Zum neuen Parlamentspräsidenten (Ceann Comhairle) wurde der frühere FG-Minister Seán Barrett gewählt. Außerdem haben sich 16 der unabhängigen Abgeordneten zu einer "technischen Gruppe“ zusammengeschlossen, um ihre Redezeiten im Dáil erhöhen zu können. ${ }^{41}$ Bei der ersten Kabinettssitzung wurde eine Gehaltskürzung des Kabinetts beschlossen. Die Bezüge des Taoiseach wurden um 14.000 auf 200.000 Euro gekürzt, die seines Stellvertreters um 13.000 auf 184.000 Euro, das Gehalt der Minister sinkt um 12.000 auf 169.000 Euro und das der Juniorminister um 9.000 auf 130.000 Euro. ${ }^{42}$

Regionale und Gender-Aspekte haben bei der Regierungsbildung keine Rolle gespielt. Zwei Ministerinnen entsprechen einem Anteil von 13,3 Prozent im Kabinett. Allerdings ist der Anteil weiblicher Dáil-Mitglieder mit 15 Prozent (25) nicht viel höher. Bezieht man auch die Staatssekretäre mit ein, liegt der Frauenanteil bei 20 Prozent (sechs Personen). Von den 15 Ministern kommen neun aus Dublin. Die insgesamt 30 Regierungsämter entsprechen einem Anteil von 18 Prozent des Dáil beziehungsweise von 26,5 Prozent der 113 FG- und Labour-Abgeordneten. Die Regelung, dass Minister auch Mitglieder des Parlaments sein müssen, schränkt neben der Praxis der Doppelmandatierung auf lokaler und nationaler Ebene den Rekrutierungspool deutlich ein. ${ }^{43}$

\section{Ausblick: Ende des dominierten Parteiensystems?}

Seit Ende der 1990er Jahre ist eine sukzessive Stärkung unabhängiger Kandidaten zu beobachten, der eine Erosion der Stimmenanteile von FF und FG von zusammen über 80 Prozent bis Anfang der 1980er Jahre auf gegenwärtig nur noch 57 Prozent gegenübersteht. Da

40 Mit der Transaktionskostentheorie ist davon auszugehen, dass das so genannte Kreuzstichverfahren die Transaktionskosten der „Überwachung“ der Vertragstreue des Vertragspartners senkt und dadurch die Überlebensdauer der Koalition erhöht, vgl. Thomas Saalfeld, Koalitionsstabilität in 15 europäischen Demokratien von 1945 bis 1999: Transaktionskosten und Koalitionsmanagement, in: ZParl, 38. Jg. (2007), H. 1, S. 180 - 206, S. 192.

41 Vgl. Stephen Collins, Momentous Day for 31st Dáil as Kenny Elected Taoiseach, in: The Irish Times vom 10. März 2011, http://www.irishtimes.com/newspaper/frontpage/2011/0310/ 1224291780303.html (Abruf am 11. April 2011).

42 Vgl. ebenda.

43 Der lokaler Klientelismus der irischen Abgeordneten, die sich traditionell primär ihrem Wahlkreis verpflichtet fühlen, ist durch das Wahlsystem der übertragbaren Einzelstimme fest im politischen System verankert. Premier Kenny hat seine Minister bei der Regierungsbildung darauf verpflichtet, in den ersten 100 Tagen keinen Wahlkreispflichten nachzukommen, um sich ganz auf ihr Amt konzentrieren zu können; vgl. Johannes Leithäuser, a.a.O. (Fn. 6). Darin zeigt sich wieder der Professionalisierungsdruck der irischen politischen Eliten, die (ähnlich der Schweiz) trotz Globalisierung und Wirtschaftskrise das Milizprinzip und den lokalen Bezug hoch halten. Die im Koalitionsvertrag angekündigte Weiterentwicklung des Ausschusswesens dürfte hierzu einen Beitrag leisten. Auch dass die Formulierung strategischer Politikziele in Irland stark den Beamten und Experten des vorpolitischen Raumes überlassen wurde, wird sich vermutlich ändern. Angesichts der aktuellen Krise dürfte eine Politisierung eingetreten und die Politik stärker direkten Einfluss nehmen. 
angesichts dieser Entwicklung der Disproportionalitätseffekt für „manufactured majorities“ einzelner Parteien kaum ausreichen wird, werden Koalitionsregierungen und politisches „bargaining“ an Bedeutung gewinnen. Dass die Labour Party bislang primär mit Fine Gael koaliert hat, ist weniger auf programmatische Nähe als auf die arithmetische Konstellation eines dominierten Parteiensystems zurückzuführen. Dennoch scheint die neue Regierungskoalition in Irland (ähnlich wie die Große Koalition in Deutschland) nicht nur aus der Not heraus entstanden, sondern auch zum Erfolg entschlossen zu sein. Sobald sich erste positive Ergebnisse bei der Erholung der Wirtschaft zeigen sollten, könnte allerdings der wahrgenommene Problemdruck und damit die wichtigste externe Bedingung interner Kohäsion nachlassen. ${ }^{44}$ Dann wird es insbesondere auf die Institutionen des Koalitionsmanagements ankommen, um Konflikte aufzufangen. ${ }^{45}$ Zwar wird im Koalitionsvertrag davon gesprochen, dass beide Parteien sich den Aufgaben „with a combination of humility and hope, underscored with an absolute resolve to bring the change the people so clearly demand" (S. 3) zuwenden; Regelungen für den Konfliktfall, wie etwa ein Koalitionsausschuss, werden aber nicht verabredet. Dadurch werden zum einen formelle Strukturen wie Kabinettssitzungen, zum anderen informelle Strukturen, zum Beispiel „Sofa-Government “ ${ }^{46}$, aufgewertet.

Langfristig wird für die Entwicklung des irischen Parteiensystems entscheidend sein, ob die strukturelle Mehrheitspartei Fianna Fáil wieder zur alten Stärke zurückfinden wird, was im Zuge der europaweit zu beobachtenden Prozesse der Erosion traditioneller Parteibindungen und parteiensystemischer Dominanzstrukturen (etwa auch in Großbritannien) nicht zwangsläufig zu erwarten ist. Die Annahme, dass Drittparteien und Unabhängige an Einfluss gewinnen werden, wird auch durch die Entwicklung der Mitgliederzahlen gestützt. Während die beiden großen Parteien Fianna Fáil und Fine Gael in den letzten circa 20 Jahren etwa 30 bis 40 Prozent ihrer Mitglieder verloren haben, ist deren Zahl bei Labour moderat gestiegen (von circa 6.500 im Jahre 1985 auf circa 7.000 im Jahr 2008). ${ }^{47}$ Nach der Selbstauflösung der Progressive Democrats 2008 könnte Labour möglicherweise eine entscheidende Kraft für zukünftig notwendige Koalitionsregierungen werden. Möglich ist aber auch, dass sich neue Koalitionsmuster unter Einbindung von Sinn Féin oder vermehrt Minderheitsregierungen herausbilden. Bei der Einbindung von Unabhängigen gibt es inzwischen eine Reihe von Fällen, in denen diese sich ihre Zustimmung mit umfangreichen Investitionen in ihren Wahlkreisen erkauft haben. ${ }^{48}$ Ihr Einfluss auf zukünftige Regierungen könnte also deutlich steigen.

44 Eine eher symbolische Integrationsfunktion dürfte die gemeinsame Forderung einer Nachverhandlung der Bedingungen des EU/IWF-Rettungspaketes gehabt haben, die unter anderem von Frankreich mit der Forderung nach höheren Unternehmenssteuern in Irland gekontert wurde. Vgl. Marcus Theurer, Die Grüne Insel ist gar keine Steueroase, in: FAZ vom 22. März 2011, S. 12.

45 Vgl. Thomas Saalfeld, a.a.O. (Fn. 2), S. 240.

46 Für Großbritannien vgl. Thomas Krumm / Thomas Noetzel, Das Regierungssystem Großbritanniens, München 2006, S. 175.

47 Vgl. Jürgen Elvert, a.a.O. (Fn. 14), S. 325. Die Struktur der Parteimitglieder ist bislang kaum untersucht. Eine Ausnahme stellen Michael Gallagher und Michael Marsh (Party Membership in Ireland: The members of Fine Gael, in: Party Politics, 10. Jg. (2004), H. 4, S. 407 - 425) dar, die den hohen Anteil langjähriger Parteimitglieder und die Rolle persönlicher Netzwerke hervorheben.

48 Vgl. Maura Adshead/ Jonathan Tonge, a.a.O. (Fn. 1), S. 100. 